\title{
DETERMINANTS OF SOME PENTADIAGONAL MATRICES
}

\author{
LÁSZLÓ LOSONCZI
}

\begin{abstract}
In this paper we consider pentadiagonal $(n+1) \times(n+1)$ matrices with two subdiagonals and two superdiagonals at distances $k$ and $2 k$ from the main diagonal where $1 \leq k<2 k \leq n$. We give an explicit formula for their determinants and also consider the Toeplitz and "imperfect" Toeplitz versions of such matrices. Imperfectness means that the first and last $k$ elements of the main diagonal differ from the elements in the middle. Using the rearrangement due to Egerváry and Szász we also show how these determinants can be factorized.
\end{abstract}

\section{INTRODUCTION}

Matrices have a special space in mathematics. Their theory is still actively researched and used by almost every mathematician and by several scientists working in various areas. The research on multidiagonal, in particular tridiagonal and pentadiagonal matrices, intensified in the past years. These matrices have important applications in optimization problems [3], autoregression modelling [26], approximation theory [23], Gauss-Markov random processes [2], orthogonal polynomials, solving elliptic and parabolic PDE's with finite difference methods [10], inequalities (quadratic, Wirtinger, Opial's type) [4], [18].

Let $n, k$ be given positive integers with $1 \leq k<2 k \leq n$ and denote by $\mathcal{M}_{n}$ the set of $n \times n$ complex matrices. Consider the pentadiagonal matrix $A_{0}=\left(a_{i j}\right) \in \mathcal{M}_{n+1}$ where for $i, j=0,1, \ldots, n$,

$$
a_{i j}= \begin{cases}L_{j} & \text { if } j-i=-2 k, \\ l_{j} & \text { if } j-i=-k, \\ d_{i} & \text { if } j-i=0, \\ r_{i} & \text { if } j-i=k, \\ R_{i} & \text { if } j-i=2 k, \\ 0 & \text { otherwise. }\end{cases}
$$

$A_{0}$ has two subdiagonals and two superdiagonals at distances $k$ and $2 k$ from the main diagonal. Notice that the numbering of entries starts with zero. We also use $A_{n+1, k, 2 k}(\mathbf{L}, \mathbf{l}, \mathbf{d}, \mathbf{r}, \mathbf{R})$ to denote the matrix $A_{0}$ and $D_{0}=D_{n+1, k, 2 k}(\mathbf{L}, \mathbf{l}, \mathbf{d}, \mathbf{r}, \mathbf{R})$ to denote its determinant where the diagonal vectors $\mathbf{L}, \mathbf{l}, \mathbf{d}, \mathbf{r}, \mathbf{R}$ are defined by

$$
\begin{aligned}
& \mathbf{L}=\left(L_{0}, \ldots, L_{n-2 k}\right) \quad \mathbf{l}=\left(l_{0}, \ldots, l_{n-k}\right) \\
& \mathbf{d}=\left(d_{0}, \ldots, d_{n}\right) \\
& \mathbf{R}=\left(R_{0}, \ldots, R_{n-2 k}\right) \quad \mathbf{r}=\left(r_{0}, \ldots, r_{n-k}\right)
\end{aligned}
$$

If $\mathbf{L}, \mathbf{R}$ are zero vectors then our matrix becomes a tridiagonal one denoted by $A_{n+1, k}(\mathbf{l}, \mathbf{d}, \mathbf{r})$ and its determinant by $D_{n+1, k}(\mathbf{l}, \mathbf{d}, \mathbf{r})$.

We shall call $A_{n+1, k, 2 k}(\mathbf{L}, \mathbf{l}, \mathbf{d}, \mathbf{r}, \mathbf{R})$ a (general) $k, 2 k$-pentadiagonal matrix while $A_{n+1, k}(\mathbf{l}, \mathbf{d}, \mathbf{r})$ will be termed as $k$-tridiagonal.

Date: May 21, 2021.

2020 Mathematics Subject Classification. 15A15, 15B99, 65F40, 15A42.

Key words and phrases. Toeplitz matrix, pentadiagonal, tridiagonal matrices. 
In case of Toeplitz pentadiagonal matrices the diagonal vectors are constant vectors, i.e.

$$
\begin{aligned}
\mathbf{L} & =(L, \ldots, L), \mathbf{R}=(R, \ldots, R) \in \mathbb{R}^{n+1-2 k}, \\
\mathbf{l} & =(l, \ldots, l), \quad \mathbf{r}=(r, \ldots, r) \in \mathbb{R}^{n+1-k}, \\
\mathbf{d} & =(d, \ldots, d) \in \mathbb{R}^{n+1},
\end{aligned}
$$

and for the matrix and its determinant the notations $A_{n+1, k, 2 k}(L, l, d, r, R)$ and $D_{n+1, k, 2 k}(L, l, d, r, R)$ will be used.

Marr and Vineyard [20]) have shown that the product of two 1-tridiagonal Toeplitz matrix is an imperfect Toeplitz matrix $A_{n+1,1,2}^{(\alpha, \beta)}$ which is related to the corresponding Toeplitz matrix by a two-step recursion. Imperfectness means that the main diagonal is changed from $(d, \ldots, d) \in \mathbb{R}^{n+1}$ to

$$
(\underbrace{d-\alpha, \ldots, d-\alpha}_{k}, \underbrace{d, \ldots, d}_{n+1-2 k}, \underbrace{d-\beta, \ldots, d-\beta}_{k}) \in \mathbb{R}^{n+1}
$$

where $\alpha, \beta$ are given reals. They found the determinants of Toeplitz and imperfect Toeplitz 1,2pentadiagonal matrices in terms of Chebyshev polynomials of the second kind. A similar approach was used in [27] to find a formula for the inverse of a 1,2-pentadiagonal Toeplitz matrix. Imperfect $k, 2 k$-pentadiagonal Toeplitz matrices will be denoted by $A_{n+1, k, 2 k}^{(\alpha, \beta)}(L, l, d, r, R)$, their determinants by $D_{n+1, k, 2 k}^{(\alpha, \beta)}(L, l, d, r, R)$.

A number of papers studied general 1,2-pentadiagonal matrices and their Toeplitz versions. Algorithms and recursive formulas were found for their determinants [24], [15], [11], [17] and inverses [16]. Explicit formulas were found for the determinants of symmetric [14] skew-symmetric [13] and general [17] Toeplitz 1,2-pentadiagonal matrices. Perhaps the first appearance of $k, \ell$-pentadiagonal (Toeplitz Hermitian) matrices (where the distances of the sub- and superdiagonals from the main one are $k$ and $\ell$ ) was in Egerváry and Szász [4] with $k+\ell=n+1$, while $k$-tridiagonal matrices appeared first in [12]. In [19] new sub/superdiagonals were added. A graph theoretical approach can be found in [5,7] and some extensions in [21,25]. An exhaustive list of recent references is given in the survey [6].

In spite of the large numbers of papers on pentadiagonal matrices there are only few of them in which determinants are given in terms of the entries.

In [8] we developed a method to reduce the determinant of $k, \ell$-pentadiagonal matrices to tridiagonal determinants provided that $k+\ell \geq n+1$. If $k \geq(n+1) / 3$ then by this method in [9] we determined the determinants of general, Toeplitz and imperfect Toeplitz $k, 2 k$-pentadiagonal matrices. We proved (among others)

Theorem 1. Assuming $(n+1) / 3 \leq k \leq n / 2$ the determinant $D_{n+1, k, 2 k}(\mathbf{L}, \mathbf{l}, \mathbf{d}, \mathbf{r}, \mathbf{R})$ of the general $k, 2 k$-pentadiagonal matrix is

$$
\prod_{j=0}^{n-2 k}\left(d_{j} d_{j+k} d_{j+2 k}-d_{j} l_{j+k} r_{j+k}-L_{j} R_{j} d_{j+k}-l_{j} r_{j} d_{j+2 k}+l_{j} R_{j} l_{j+k}+r_{j} L_{j} r_{j+k}\right) \prod_{j=n+1-2 k}^{k-1}\left(d_{j} d_{j+k}-l_{j} r_{j}\right) .
$$

If the condition $k+\ell \geq n+1$ is not satisfied then the method given in [8] is not applicable in general. However, if $\ell=2 k$ then with some modification it is applicable. In Section 2 we develop this modification and extend Theorem 1 to the case when the restriction $(n+1) / 3 \leq k$ is dropped. Finally in Section 3 we show how the determinants $D_{n+1, k, 2 k}(L, l, d, r, R)$ can be factorized and also discuss the case of Toeplitz determinants. 


\section{REDUCTION OF GENERAL $k, 2 k$-PENTADIAGONAL DETERMINANTS TO TRIDIAGONAL ONES}

Our starting point is the matrix $A_{0}=A_{n+1, k, 2 k}(\mathbf{L}, \mathbf{l}, \mathbf{d}, \mathbf{r}, \mathbf{R})$ where $1 \leq k<2 k \leq n$. Let $n+1=$ $k q+p$ where $0 \leq p<k$ and suppose that $k<(n+1) / 3$ (or $q \geq 3$ ).

In the first process we multiply $A_{0}$ by four suitable matrices such that in the product matrix the first $k$ rows and columns contains only zeros except the diagonal and in the lower $(n+1-k) \times(n+1-k)$ block we get a pentadiagonal matrix whose structure is similar to that of $A_{0}$.

(i) Let $B_{1}^{(l)} \in \mathcal{M}_{n+1}$ with entries

$$
b_{i j}^{(1, l)}=\left\{\begin{aligned}
1 & \text { if } i=j, \\
-l_{j} / d_{j} & \text { if } i=k, \ldots, 2 k-1, j=i-k, \\
0 & \text { otherwise }
\end{aligned}\right.
$$

and multiply $A_{0}$ from the left by $B_{1}^{(l)}$. The effect of this multiplication is the same as multiplying the rows $0, \ldots, k-1$ of $A_{0}$ by the numbers $-l_{0} / d_{0}, \ldots,-l_{k-1} / d_{k-1}$ and adding them to the rows $k, \ldots, 2 k-1$, respectively. The elements $l_{0}, \ldots, l_{k-1}$ of the $k$ th subdiagonal disappear and the diagonal elements $d_{k}, \ldots, d_{2 k-1}$ change to

$$
d_{j}^{(1)}:=d_{j}-l_{j-k} r_{j-k} / d_{j-k} \quad \text { for } j=k, \ldots, 2 k-1 .
$$

The $2 k$ th superdiagonal remains unchanged, however all its elements $R_{0}, \ldots, R_{k-1}$ multiplied one by one by $l_{0} / d_{0}, \ldots,-l_{k-1} / d_{k-1}$, respectively move down by $k$ units and added to $r_{k}, \ldots, r_{2 k-1}$ thus these elements change to

$$
r_{j}^{(1)}=r_{j}-R_{j-k} l_{j-k} / d_{j-k}, \quad \text { if } j=k, \ldots, 2 k-1 .
$$

Please note that the position of entries $-l_{j} / d_{j}(j=0, \ldots, k-1)$ in the matrix $B_{1}^{(l)}$ is the same as the position of $l_{j}(j=0, \ldots, k-1)$ in the matrix $A_{n+1, k, \ell}(\mathbf{L}, \mathbf{l}, \mathbf{d}, \mathbf{r}, \mathbf{R})$, the position of the entries we want to eliminate. The matrices $B_{1}^{(r)}, B_{1}^{(L)}, B_{1}^{(R)}$ in the following multiplications also have similar structures.

(ii) Multiply $B_{1}^{(l)} A_{0}$ from the right by $B_{1}^{(r)} \in \mathcal{M}_{n+1}$ with entries

$$
b_{i j}^{(1, r)}:=\left\{\begin{aligned}
1 & \text { if } i=j, \\
-r_{i} / d_{i} & \text { if } i=0, \ldots, k-1 ; j=i+k, \\
0 & \text { otherwise }
\end{aligned}\right.
$$

The effect of this, is multiplication of the columns $0, \ldots, k-1$ of $B_{1}^{(l)} A_{0}$ by $-r_{0} / d_{0}, \ldots,-r_{k-1} / d_{k-1}$ and addition of these products to the columns $k, \ldots, 2 k-1$, respectively. The elements $r_{0}, \ldots, r_{k-1}$ in these columns disappear the diagonal elements remain unchanged.

The $2 k$ th subdiagonal remains unchanged, however its elements $L_{0}, \ldots, L_{k-1}$ multiplied one by one by $-r_{0} / d_{0}, \ldots,-r_{k-1} / d_{k-1}$ respectively move to the right by $k$ units and added to the elements $l_{2 k}, \ldots, l_{3 k-1}$, hence these elements change to

$$
l_{j}^{(1)}=l_{j}-L_{j-k} r_{j-k} / d_{j-k}, \quad \text { if } j=2 k, \ldots, 3 k-1 .
$$

(iii) Next multiply $B_{1}^{(l)} A_{0} B_{1}^{(r)}$ from the left by $B_{1}^{(L)} \in \mathcal{M}_{n+1}$ with entries

$$
b_{i j}^{(1, L)}:= \begin{cases}1 & \text { if } i=j, \\ -L_{j} / d_{j} & \text { if } i=2 k, \ldots, 3 k-1 ; j=i-2 k, \\ 0 & \text { otherwise }\end{cases}
$$


The effect of this on the matrix $B_{1}^{(l)} A_{0} B_{1}^{(r)}$ is multiplication of its rows $0, \ldots, n-2 k$ by $-L_{0} / d_{0}, \ldots$, $-L_{n-2 k} / d_{n-2 k}$ and adding these products to the rows $2 k, \ldots, 3 k-1$, respectively. The elements $L_{0}, \ldots, L_{k-1}$ of the $2 k$ th subdiagonal disappear, the elements $d_{2 k}, \ldots, d_{3 k-1}$ of the main diagonal change to

$$
d_{j}^{(1)}=d_{j}-L_{j-2 k} R_{j-2 k} / d_{j-2 k}, \quad \text { for } j=2 k, \ldots, 3 k-1 .
$$

(iv) Finally multiply $B_{1}^{(L)} B_{1}^{(l)} A_{0} B_{1}^{(r)}$ from the right by $B_{1}^{(R)} \in \mathcal{M}_{n+1}$ with entries

$$
b_{i j}^{(1, R)}:= \begin{cases}1 & \text { if } i=j, \\ -R_{i} / d_{i} & \text { if } i=0, \ldots, k-1 ; j=i+2 k, \\ 0 & \text { otherwise }\end{cases}
$$

The effect of this on the matrix $B_{1}^{(L)} B_{1}^{(l)} A_{0} B_{1}^{(r)}$ is multiplication of its columns $0, \ldots, k-1$ by $-R_{0} / d_{0}, \ldots,-R_{k-1} / d_{k-1}$ and adding these products to the columns $2 k, \ldots, 3 k-1$ respectively. The elements $R_{0}, \ldots, R_{k-1}$ vanish and the main diagonal does not change.

With this the first process ended. The matrices $B_{1}^{(L)}, B_{1}^{(l)}, B_{1}^{(r)}, B_{1}^{(R)}$ are the same as in [8, 9] however the transformation rules for the entries are different. In those papers during the first (and subsequent) processes the diagonal vectors $\mathbf{l}, \mathbf{r}$ just shortened while the diagonal vectors $\mathbf{L}, \mathbf{R}$ transformed. Here just the opposite happened: the diagonals $\mathbf{L}, \mathbf{R}$ shortened and $\mathbf{l}, \mathbf{r}$ transformed.

The results of the first process are summarized in

Theorem 2. Suppose that $1 \leq k \leq 2 k \leq n, k<(n+1) / 3$. Then

$$
A_{1}:=B_{1}^{(L)} B_{1}^{(l)} A_{0} B_{1}^{(r)} B_{1}^{(R)}=E_{1} \bigoplus A_{1}^{*}
$$

where $E_{1} \in \mathcal{M}_{k}$ is a diagonal matrix with diagonal elements $d_{0}, \ldots, d_{k-1}$ and

$$
A_{1}^{*}=A_{n+1-k, k, 2 k}\left(\mathbf{L}^{(\mathbf{1})}, \mathbf{l}^{(\mathbf{1})}, \mathbf{d}^{(\mathbf{1})}, \mathbf{r}^{(\mathbf{1})}, \mathbf{R}^{(\mathbf{1})}\right) .
$$

is a $k, 2 k$-pentadiagonal matrix with main and other diagonal vectors (called first iterated diagonals)

$$
\begin{aligned}
\mathbf{L}^{(1)} & =\left(L_{k}, \ldots, L_{n-2 k}\right) \in \mathbb{R}^{n+1-3 k} & \mathbf{R}^{(1)} & =\left(R_{k}, \ldots, R_{n-2 k}\right) \in \mathbb{R}^{n+1-3 k} \\
\mathbf{l}^{(1)} & =\left(l_{k}^{(1)}, \ldots, l_{n-k}^{(1)}\right) \in \mathbb{R}^{n+1-2 k} & \mathbf{r}^{(1)} & =\left(r_{k}^{(1)}, \ldots, r_{n-k}^{(1)}\right) \in \mathbb{R}^{n+1-2 k} \\
\mathbf{d}^{(1)} & =\left(d_{k}^{(1)}, \ldots, d_{n}^{(1)}\right) \in \mathbb{R}^{n+1-k} & &
\end{aligned}
$$

where

$$
\begin{aligned}
& l_{j}^{(1)}= \begin{cases}l_{j}-L_{j-k} r_{j-k} / d_{j-k} & (j=k, \ldots, 2 k-1), \\
l_{j} & (j=2 k, \ldots, n-k)\end{cases} \\
& r_{j}^{(1)}= \begin{cases}r_{j}-R_{j-k} l_{j-k} / d_{j-k} & (j=k, \ldots, 2 k-1) \\
r_{j} & (j=2 k, \ldots, n-k)\end{cases}
\end{aligned}
$$

and

$$
d_{j}^{(1)}= \begin{cases}d_{j}-l_{j-k} r_{j-k} / d_{j-k} & (j=k, \ldots, 2 k-1), \\ d_{j}-L_{j-2 k} R_{j-2 k} / d_{j-2 k} & (j=2 k, \ldots, 3 k-1), \\ d_{j} & (j=3 k, \ldots, n),\end{cases}
$$

Proof. From the description of the four matrix multiplication (9) follows while (3), (5) show the correctness of (10) and (2), (7) show that (11) is valid. 
For the convenience of later calculations all letters $l_{i}, d_{i}, r_{i}$ referring to the original matrix will be labeled with superscripts ${ }^{(0)}$ but occasionally we omit this label. We omit from this labeling $L_{i}, R_{i}$ since these numbers did not change during our process.

Define for $s=1,2, \ldots, q-1$ the matrices of $\mathcal{M}_{n+1}$ (for $s=1$ these coincide with the matrices (1), (4), (6), (8)) which we use in further calculations by

$$
\begin{aligned}
& B_{s}^{(l)}=\left(b_{i j}^{(s, l)}\right)= \begin{cases}1 & \text { if } i=j, \\
-l_{j}^{(s-1)} / d_{j}^{(s-1)} & \text { if } i=s k, \ldots,(s+1) k-1 ; j=i-k, \\
0 & \text { otherwise, }\end{cases} \\
& B_{s}^{(r)}=\left(b_{i j}^{(s, r)}\right)= \begin{cases}1 & \text { if } i=j, \\
-r_{i}^{(s-1)} / d_{i}^{(s-1)} & \text { if } i=(s-1) k, \ldots, s k-1 ; j=i+k, \\
0 & \text { otherwise, }\end{cases} \\
& B_{s}^{(L)}=\left(b_{i j}^{(s, L)}\right)= \begin{cases}1 & \text { if } i=j, \\
-L_{j}^{(s-1)} / d_{j}^{(s-1)} & \text { if } i=(s+1) k, \ldots,(s+2) k-1 ; j=i-2 k, \\
0 & \text { otherwise, }\end{cases} \\
& B_{s}^{(R)}=\left(b_{i j}^{(s, R)}\right)= \begin{cases}1 & \text { if } i=j, \\
-R_{i}^{(s-1)} / d_{i}^{(s-1)} & \text { if } i=(s-1) k, \ldots, s k-1 ; j=i+2 k, \\
0 & \text { otherwise. }\end{cases}
\end{aligned}
$$

We define the first and second matrices for $s=q$ too, but in this case the index sets are restricted to $i=q k, \ldots, n ; j=i-k$ and $i=(q-1) k, \ldots, n-k ; j=i+k$ respectively, if $p=0$ then both sets are empty and the first and second matrices are degenerate to unit matrices. Similarly for the third and fourth matrices with $s=q-1$ the index domains are $i=q k, \ldots, n ; j=i-2 k$ and $i=(q-2) k, \ldots, n-2 k ; j=i+2 k$ respectively and if $p=0$ then both sets are empty and the third and fourth matrices are degenerate to unit matrices.

In the above definitions the quantities $l_{j}^{(s-1)}, r_{j}^{(s-1)}, d_{j}^{(s-1)}$ can be obtained inductively by continuing the iterations of (10), (11) for $s=1,2, \ldots,(q-2, q-1, q)$ as follows

$$
\begin{aligned}
& l_{j}^{(s)}= \begin{cases}l_{j}^{(s-1)}-L_{j-k} r_{j-k}^{(s-1)} / d_{j-k}^{(s-1)} & (j=s k, \ldots,(s+1) k-1), \\
l_{j} & (j=(s+1) k, \ldots, n-k)\end{cases} \\
& r_{j}^{(s)}= \begin{cases}r_{j}^{(s-1)}-R_{j-k} l_{j-k}^{(s-1)} / d_{j-k}^{(s-1)} & (j=s k, \ldots,(s+1) k-1), \\
r_{j} & (j=(s+1) k, \ldots, n-k)\end{cases}
\end{aligned}
$$

and

$$
d_{j}^{(s)}= \begin{cases}d_{j}^{(s-1)}-l_{j-k}^{(s-1)} r_{j-k}^{(s-1)} / d_{j-k}^{(s-1)} & (j=s k, \ldots,(s+1) k-1), \\ d_{j}^{(s-1)}-L_{j-2 k} R_{j-2 k} / d_{j-2 k}^{(s-1)} & (j=(s+1) k, \ldots,(s+2) k-1), \\ d_{j} & (j=(s+2) k, \ldots, n),\end{cases}
$$

These definitions are valid if $s<q-2$. We put the last three values $q-2, q-1, q$ of $s$ in parenthesis since for them the definitions should be modified. For these values of $s$ the index groups in (12), (13) may run out of their ranges $j=s k, \ldots, n-k$ and $j=s k, \ldots, n$ therefore cannot be defined, or the index groups may be restricted.

For example (13) is valid for $s \leq q-2$, for $s=q-1$ the second index group should be restricted to $j=q k, \ldots, n$ if $p>0$ and empty if $p=0$ with empty third index group. For $s=q$ the diagonal 
elements $d_{j}^{(q)}$ can be defined only if $p>0$, in this case for $s=q$ in (13) the first index group should be restricted to $j=q k, \ldots, n$ with empty second and third index groups.

In the second process we calculate the product $A_{2}:=B_{2}^{(L)} B_{2}^{(l)} A_{1} B_{2}^{(r)} B_{2}^{(R)}$ then we continue similarly $s-2(s<q-1)$ times to get

$$
A_{s}:=\left(\prod_{j=1}^{j=s} B_{s+1-j}^{(L)} B_{s+1-j}^{(l)}\right) A_{0}\left(\prod_{j=1}^{j=s} B_{j}^{(r)} B_{j}^{(R)}\right) .
$$

For $A_{s}$ we have the decomposition

$$
A_{s}=E_{s k} \bigoplus A_{s}^{*}
$$

where $E_{s k} \in \mathcal{M}_{s k}$ is a diagonal matrix with diagonal elements

$$
d_{0}, \ldots, d_{k-1}, d_{k}^{(1)}, \ldots, d_{2 k-1}^{(1)}, \ldots, d_{(s-1) k}^{(s-1)}, \ldots, d_{s k-1}^{(s-1)}
$$

and

$$
A_{s}^{*}=A_{n+1-s k, k, 2 k}\left(\mathbf{L}^{(s)}, \mathbf{l}^{(s)}, \mathbf{d}^{(s)}, \mathbf{r}^{(s)}, \mathbf{R}^{(s)}\right)
$$

with

$$
\begin{array}{rlrl}
\mathbf{L}^{(s)} & =\left(L_{s k}, \ldots, L_{n-2 k}\right) \in \mathbb{R}^{n+1-(s+2) k} & \mathbf{R}^{(s)} & =\left(R_{s k}, \ldots, R_{n-2 k}\right) \in \mathbb{R}^{n+1-(s+2) k} \\
\mathbf{l}^{(s)} & =\left(l_{s k}^{(s-1)}, \ldots, l_{n-k}^{(s-1)}\right) \in \mathbb{R}^{n+1-(s+1) k} & \mathbf{r}^{(s)}=\left(r_{s k}^{(s-1)}, \ldots, r_{n-k}^{(s-1)}\right) \in \mathbb{R}^{n+1-(s+1) k} \\
\mathbf{d}^{(s)} & =\left(d_{s k}^{(s-1)}, \ldots, d_{n}^{(s-1)}\right) \in \mathbb{R}^{n+1-s k} & &
\end{array}
$$

and the elements of these iterated diagonals are given by (12), (13).

For $s=q-2$ the dimension of the vectors $\mathbf{L}^{(s)}, \mathbf{R}^{(s)}$ is $p$. This means that if $p=0$ then $A_{q-2}^{*}$ is tridiagonal, and if $p>0$ then $A_{q-1}^{*}$ is tridiagonal. Therefore the cases $p=0$ and $p>0$ should be treated differently.

Theorem 3. Let $1 \leq k<2 k \leq n, n+1=k q$ (i.e. $p=0) q>3$. Then

$$
A_{q-1}:=B_{q-2}^{(l)} A_{q-2} B_{q-2}^{(r)}=E_{q k} \in \mathcal{M}_{n+1}
$$

is a diagonal matrix with diagonal elements

$$
d_{0}, \ldots, d_{k-1}, d_{k}^{(1)}, \ldots, d_{2 k-1}^{(1)}, \ldots, d_{(q-1) k}^{(q-1)}, \ldots, d_{q k-1}^{(q-1)}
$$

defined by (13).

If $p>0$ then multiplying $A_{q-1}$ from the left by $B_{q}^{(l)}$ and the from the right by $B_{q}^{(r)}$ we get a diagonal matrix.

Theorem 4. Let $1 \leq k<2 k \leq n, n+1=k q+p, 0 \leq p<k, q \geq 3$. Then

$$
A_{q}:=B_{q}^{(l)} A_{q-1} B_{q}^{(r)}=E_{q k+p} \in \mathcal{M}_{n+1}
$$

is a diagonal matrix with diagonal elements

$$
d_{0}, \ldots, d_{k-1}, d_{k}^{(1)}, \ldots, d_{2 k-1}^{(1)}, \ldots, d_{(q-1) k}^{(q-1)}, \ldots, d_{q k-1}^{(q-1)}, d_{q k}^{(q)}, \ldots, d_{q k+p-1}^{(q)}
$$

defined by (13).

For the determinant we have 
Theorem 5. Let $1 \leq k<2 k \leq n, n+1=k q+p, 0 \leq p<k$, and suppose that either $q=3, p>0$, or $q>3$. Then the determinant of $A_{n+1, k, 2 k}(\mathbf{L}, \mathbf{l}, \mathbf{d}, \mathbf{r}, \mathbf{R})$ is

$$
\begin{aligned}
D_{n+1, k, 2 k}(\mathbf{L}, \mathbf{l}, \mathbf{d}, \mathbf{r}, \mathbf{R}) & =\prod_{j=0}^{k-1} d_{j}^{(0)} d_{j+k}^{(1)} \cdots d_{j+(q-1) k}^{(q-1)} \prod_{j=0}^{p-1} d_{j+q k}^{(q)} \\
& =\prod_{j=0}^{p-1} d_{j}^{(0)} d_{j+k}^{(1)} \cdots d_{j+q k}^{(q)} \prod_{j=p}^{k-1} d_{j}^{(0)} d_{j+k}^{(1)} \cdots d_{j+(q-1) k}^{(q-1)}
\end{aligned}
$$

where $d_{j}^{(s)}(s=1, \ldots, q ; j=s k, \ldots, n)$ are defined by (11), (13) and $\prod_{j=0}^{-1}:=1$.

To express (14) in terms of the entries of $A_{0}$ is quite complicated if $q$ is large. Next we do this calculation if $q=3$.

For $j=0, \ldots, k-1$ we have

$$
d_{j}^{(0)} d_{j+k}^{(1)}=d_{j}\left(d_{j+k}-\frac{l_{j} r_{j}}{d_{j}}\right)=d_{j} d_{j+k}-l_{j} r_{j}
$$

and

$$
\begin{aligned}
d_{j+2 k}^{(2)} & =d_{j+2 k}^{(1)}-\frac{l_{j+k}^{(1)} r_{j+k}^{(1)}}{d_{j+k}^{(1)}}=d_{j+2 k}-\frac{L_{j} R_{j}}{d_{j}}-\frac{\left(l_{j+k}-\frac{L_{j} r_{j}}{d_{j}}\right)\left(r_{j+k}-\frac{R_{j} l_{j}}{d_{j}}\right)}{d_{j+k}-\frac{l_{j} r_{j}}{d_{j}}} \\
& =\frac{d_{j} d_{j+2 k}-L_{j} R_{j}}{d_{j}}-\frac{\left(l_{j+k} d_{j}-L_{j} r_{j}\right)\left(r_{j+k} d_{j}-R_{j} l_{j}\right)}{d_{j}\left(d_{j+k} d_{j}-l_{j} r_{j}\right)} \\
& =\frac{\left(d_{j} d_{j+2 k}-L_{j} R_{j}\right)\left(d_{j+k} d_{j}-l_{j} r_{j}\right)-\left(l_{j+k} d_{j}-L_{j} r_{j}\right)\left(r_{j+k} d_{j}-R_{j} l_{j}\right)}{d_{j}\left(d_{j+k} d_{j}-l_{j} r_{j}\right)} \\
& =\frac{d_{j} d_{j+k} d_{j+2 k}-d_{j+2 k} l_{j} r_{j}-d_{j+k} L_{j} R_{j}-d_{j} l_{j+k} r_{j+k}+R_{j} l_{j} l_{j+k}+L_{j} r_{j} r_{j+k}}{d_{j} d_{j+k}-l_{j} r_{j}} \\
d_{j}^{(0)} d_{j+k}^{(1)} d_{j+2 k}^{(2)} & =d_{j} d_{j+k} d_{j+2 k}-d_{j+2 k} l_{j} r_{j}-d_{j+k} L_{j} R_{j}-d_{j} l_{j+k} r_{j+k}+R_{j} l_{j} l_{j+k}+L_{j} r_{j} r_{j+k} .
\end{aligned}
$$

If $p>0$ then for $j=0, \ldots, p$ we have to calculate $d_{j+3 k}^{(3)}$ too.

$$
d_{j+3 k}^{(3)}=d_{j+3 k}^{(2)}-\frac{l_{j+2 k}^{(2)} r_{j+2 k}^{(2)}}{d_{j+2 k}^{(2)}}=\frac{d_{j+3 k}^{(2)} d_{j+2 k}^{(2)}-l_{j+2 k}^{(2)} r_{j+2 k}^{(2)}}{d_{j+2 k}^{(2)}}
$$


where

$$
\begin{aligned}
d_{j+3 k}^{(2)} & =d_{j+3 k}^{(1)}-\frac{L_{j+k} R_{j+k}}{d_{j+k}^{(1)}}=d_{j+3 k}-\frac{L_{j+k} R_{j+k}}{d_{j+k}-\frac{l_{j} r_{j}}{d_{j}}}=\frac{d_{j} d_{j+k} d_{j+3 k}-d_{j+3 k} l_{j} r_{j}-d_{j} L_{j+k} R_{j+k}}{d_{j} d_{j+k}-l_{j} r_{j}} \\
l_{j+2 k}^{(2)} & =l_{j+2 k}^{(1)}-\frac{L_{j+k} r_{j+k}^{(1)}}{d_{j+k}^{(1)}}=l_{j+2 k}-\frac{L_{j+k}\left(r_{j+k}-\frac{R_{j} l_{j}}{d_{j}}\right)}{d_{j+k}-\frac{l_{j} r_{j}}{d_{j}}} \\
& =\frac{d_{j} d_{j+k} l_{j+2 k}-l_{j} l_{j+2 k} r_{j}-d_{j} L_{j+k} r_{j+k}+L_{j+k} l_{j} R_{j}}{d_{j} d_{j+k}-l_{j} r_{j}} \\
r_{j+2 k}^{(2)} & =r_{j+2 k}^{(1)}-\frac{R_{j+k} l_{j+k}^{(1)}}{d_{j+k}^{(1)}}=r_{j+2 k}-\frac{R_{j+k}\left(l_{j+k}-\frac{L_{j} r_{j}}{d_{j}}\right)}{d_{j+k}-\frac{l_{j} r_{j}}{d_{j}}} \\
& =\frac{d_{j} d_{j+k} r_{j+2 k}-r_{j} r_{j+2 k} l_{j}-d_{j} R_{j+k} l_{j+k}+R_{j+k} r_{j} L_{j}}{d_{j} d_{j+k}-l_{j} r_{j}}
\end{aligned}
$$

Denote by $n_{1}, n_{2}, n_{3}, n_{4}$ the numerators by $m_{1}, m_{2}, m_{3}, m_{4}$ the denominators of the above fraction forms of $d_{j+3 k}^{(2)}, d_{j+2 k}^{(2)}, l_{j+2 k}^{(2)}, r_{j+2 k}^{(2)}$ respectively, then we have that

$$
d_{j+3 k}^{(3)}=\frac{n_{1} n_{2}-n_{3} n_{4}}{\left(d_{j+k} d_{j}-l_{j} r_{j}\right)^{2}} \frac{d_{j+k} d_{j}-l_{j} r_{j}}{n_{2}}=\frac{n_{1} n_{2}-n_{3} n_{4}}{\left(d_{j+k} d_{j}-l_{j} r_{j}\right) n_{2}} .
$$

Therefore

$$
d_{j}^{(0)} d_{j+k}^{(1)} d_{j+2 k}^{(2)} d_{j+3 k}^{(3)}=n_{2} d_{j+3 k}^{(3)}=\frac{n_{1} n_{2}-n_{3} n_{4}}{d_{j+k} d_{j}-l_{j} r_{j}} .
$$

Calculating and factorizing the numerator by Maple software we obtain

$$
n_{1} n_{2}-n_{3} n_{4}=\left(d_{j+k} d_{j}-l_{j} r_{j}\right) M
$$

where

$$
\begin{aligned}
M: & =d_{j} d_{j+k} d_{j+2 k} d_{j+3 k}-d_{j} d_{j+k} l_{j+2 k} r_{j+2 k}-d_{j} d_{j+2 k} L_{j+k} R_{j+k}-d_{j} d_{j+3 k} l_{j+k} r_{j+k}-d_{j+k} d_{j+3 k} L_{j} R_{j} \\
& -d_{j+2 k} d_{j+3 k} l_{j} r_{j}+d_{j} L_{j+k} r_{j+k} r_{j+2 k}+d_{j} R_{j+k} l_{j+k} l_{j+2 k}+d_{j+3 k} L_{j} r_{j} r_{j+k}+d_{j+3 k} R_{j} l_{j} l_{j+k} \\
& +L_{j} L_{j+k} R_{j} R_{j+k}-L_{j} R_{j+k} l_{j+2 k} r_{j}-L_{j+k} R_{j} l_{j} r_{j+2 k}+l_{j} l_{j+2 k} r_{j} r_{j+2 k},
\end{aligned}
$$

therefore

$$
d_{j}^{(0)} d_{j+k}^{(1)} d_{j+2 k}^{(2)} d_{j+3 k}^{(3)}=M=M_{n, k, j}(\mathbf{L}, \mathbf{l}, \mathbf{d}, \mathbf{r}, \mathbf{R}) .
$$

Further let

$$
\begin{aligned}
& N_{n, k, j}(\mathbf{L}, \mathbf{l}, \mathbf{d}, \mathbf{r}, \mathbf{R}):=n_{2} \\
& \quad=d_{j} d_{j+k} d_{j+2 k}-d_{j+2 k} l_{j} r_{j}-d_{j+k} L_{j} R_{j}-d_{j} l_{j+k} r_{j+k}+R_{j} l_{j} l_{j+k}+L_{j} r_{j} r_{j+k}
\end{aligned}
$$

then we have 
Theorem 6. Let $1 \leq k<2 k \leq n, n+1=3 k+p, 0 \leq p<k$ then

$$
D_{n+1, k, 2 k}(\mathbf{L}, \mathbf{l}, \mathbf{d}, \mathbf{r}, \mathbf{R})=\prod_{j=0}^{p-1} d_{j}^{(0)} d_{j+k}^{(1)} d_{j+2 k}^{(2)} d_{j+3 k}^{(3)} \prod_{j=p}^{k-1} d_{j}^{(0)} d_{j+k}^{(1)} d_{j+2 k}^{(2)}
$$

$$
=\prod_{j=0}^{p-1} M_{n, k, j}(\mathbf{L}, \mathbf{l}, \mathbf{d}, \mathbf{r}, \mathbf{R}) \prod_{j=p}^{k-1} N_{n, k, j}(\mathbf{L}, \mathbf{l}, \mathbf{d}, \mathbf{r}, \mathbf{R})
$$

where $\prod_{j=0}^{-1}:=1$.

\section{3. $k, 2 k$-PENTADIAGONAL DETERMINANTS, THE TOEPLitz CASE}

In the imperfect Toeplitz case in the quantities $M_{n, k, j}, N_{n, k, j}$ of (15) we have to substitute $L_{j}=$ $L, l_{j}=l, r_{j}=r, R_{j}=R$ and

in $M_{n, k, j}$ for $j=0, \ldots, p-1$ substitute $d_{j}=d-\alpha, d_{j+k}=d, d_{j+2 k}=d, d_{j+3 k}=d-\beta$,

in $N_{n, k, j}$ for $j=p, \ldots, k-1$ substitute $d_{j}=d-\alpha, d_{j+k}=d, d_{j+2 k}=d-\beta$.

We obtain

Theorem 7. Let $1 \leq k<2 k \leq n, n+1=3 k+p, 0 \leq p<k$ then the determinant of the imperfect pentadiagonal Toeplitz matrix $A_{n+1, k, 2 k}^{(\alpha, \beta)}(L, l, d, r, R)$ is

$$
\begin{aligned}
D_{n+1, k, 2 k}^{(\alpha, \beta)} & =\left(d^{4}-(\alpha+\beta) d^{3}-(3 l r+2 L R-\alpha \beta) d^{2}+\left(2 L r^{2}+2 R l^{2}+(\alpha+\beta)(2 l r+L R)\right) d\right. \\
& \left.+L^{2} R^{2}-2 L R l r+l^{2} r^{2}+(\alpha+\beta)\left(L r^{2}+R l^{2}\right)-\alpha \beta l r\right)^{p} \\
& \cdot\left(d^{3}-(\alpha+\beta) d^{2}-(2 l r+L R-\alpha \beta) d+L r^{2}+R l^{2}+\alpha \beta l r\right)^{k-p}
\end{aligned}
$$

In the Toeplitz case we immediately get by $\alpha=\beta=0$ from the previous theorem

Theorem 8. Let $1 \leq k<2 k \leq n, n+1=3 k+p, 0 \leq p<k$ then the determinant of $A_{n+1, k, 2 k}(L, l, d, r, R)$ is

$$
\begin{aligned}
D_{n+1, k, 2 k}= & \left(d^{4}-(3 l r+2 L R) d^{2}+\left(2 L r^{2}+2 R l^{2}\right) d+L^{2} R^{2}-2 L R l r+l^{2} r^{2}\right)^{p} \\
& \cdot\left(d^{3}-(2 l r+L R) d+L r^{2}+R l^{2}\right)^{k-p} .
\end{aligned}
$$

Using a suitable rearrangement of $k, 2 k$-pentadiagonal (general or Toeplitz) matrices they can be reduced to the direct sum of 1,2-pentadiagonal (general or Toeplitz) matrices. In this way we can say more about $k, 2 k$-pentadiagonal determinants.

Let $n+1=k q+p$ where $0 \leq p<k$ and consider the permutation $\sigma$ of the integers $0,1, \ldots, n$ given by

$$
\begin{array}{rll}
\sigma(s+j(q+1))=s k+j & \text { if } s=0,1, \ldots, q ; j=0, \ldots, p-1 \\
\sigma(s+p+j q)=s k+j \quad \text { if } s=0,1, \ldots, q-1 ; j=p, \ldots, k-1
\end{array}
$$

Define the permutation matrix $P_{\sigma}=\left(p_{i j}\right)$ by

$$
p_{i j}=\left\{\begin{array}{l}
1 \text { if } j=\sigma(i) \\
0 \text { otherwise }
\end{array}\right.
$$


Then $P_{\sigma} A_{0} P_{\sigma}^{T}$ rearranges both the rows and columns of $A_{0}=A_{n+1, k, 2 k}(\mathbf{L}, \mathbf{l}, \mathbf{d}, \mathbf{r}, \mathbf{R})$ in the order of the permutation $\sigma$. Thus we obtain

$$
P_{\sigma} A_{0} P_{\sigma}^{T}=\left(\bigoplus_{j=0}^{p-1} A_{q+1,1,2}^{(j)}\right)\left(\bigoplus_{j=p}^{k-1} A_{q, 1,2}^{(j)}\right)
$$

where $A_{t+1,1,2}^{(s)}\left(\mathbf{L}_{(s)}, \mathbf{l}_{(s)}, \mathbf{d}_{(s)}, \mathbf{r}_{(s)}, \mathbf{R}_{(s)}\right)$ for $t=q, s=0,1, \ldots, p-1$ and $t=q-1, s=p, p+$ $1, \ldots, k-1$ are 1,2 -pentadiagonal matrices with diagonal vectors

$$
\begin{aligned}
\mathbf{L}_{(s)} & =\left(L_{s}, L_{s+k}, \ldots, L_{s+(t-2) k}\right), & \mathbf{R}_{(s)} & =\left(R_{s}, R_{s+k}, \ldots, R_{s+(t-2) k}\right), \\
\mathbf{l}_{(s)} & =\left(l_{s}, l_{s+k}, \ldots, l_{s+(t-1) k}\right), & \mathbf{r}_{(s)} & =\left(r_{s}, r_{s+k}, \ldots, r_{s+(t-1) k}\right), \\
\mathbf{d}_{(s)} & =\left(d_{s}, d_{s+k}, \ldots, d_{s+t k}\right) . & &
\end{aligned}
$$

We remark that $\sigma$ is the same permutation as the one used by Egerváry and Szász [4], see also N. Bebiano and S. Furtado [1] where a similar decomposition were given.

From (16) it follows immediately

Theorem 9. Let $1 \leq k<2 k \leq n$, then we have

$$
D_{n+1, k, 2 k}(\mathbf{L}, \mathbf{l}, \mathbf{d}, \mathbf{r}, \mathbf{R})=\prod_{j=0}^{p-1} D_{q+1,1,2}^{(j)} \prod_{j=p}^{k-1} D_{q, 1,2}^{(j)}
$$

where $D_{t+1,1,2}^{(s)}$ denoted the determinant of the matrix $A_{t+1,1,2}^{(s)}\left(\mathbf{L}_{(s)}, \mathbf{l}_{(s)}, \mathbf{d}_{(s)}, \mathbf{r}_{(s)}, \mathbf{R}_{(s)}\right)$.

For Toeplitz matrices we get (with the notation $B^{\oplus j}:=\underbrace{B \oplus \cdots \oplus B}_{j}$ )

$$
P_{\sigma} A_{n+1, k, 2 k}(L, l, d, r, R) P_{\sigma}^{T}=A_{q+1,1,2}(L, l, d, r, R)^{\oplus p} A_{q, 1,2}(L, l, d, r, R)^{\oplus(k-p)}
$$

as in this case $A_{t+1,1,2}^{(s)}\left(\mathbf{L}_{(s)}, \mathbf{l}_{(s)}, \mathbf{d}_{(s)}, \mathbf{r}_{(s)}, \mathbf{R}_{(s)}\right)=A_{t+1,1,2}(L, l, d, r, R)$ for $t=q, s=0,1, \ldots, p-1$ and $t=q-1, s=p, p+1, \ldots, k-1$.

For the determinants we get

$$
D_{n+1, k, 2 k}(L, l, d, r, R)=D_{q+1,1,2}(L, l, d, r, R)^{p} D_{q, 1,2}(L, l, d, r, R)^{k-p}
$$

thus it is enough to calculate the determinants of 1,2-pentadiagonal matrices. To do this we could use the iteration formulae (11), (13) (which are now considerably simpler) to find the diagonal elements then by (14) to find the determinants. However it seems easier to apply existing recursion formulae for the determinants. The six term recursion of R.A. Sweet [24] is applicable for the determinants of Toeplitz matrices but its coefficients contain fractions and are more complicated than those of the seven term recursion found by J. Jia, B. Yang, S. Li [17] thus we apply the latter.

Let $D(n+1):=D_{n+1,1,2}(L, l, d, r, R)$ for $n \geq 2$ and let $D(-2)=0, D(-1)=0, D(0)=1$, $D(1)=d, D(2)=d^{2}-l r$. For the determinant $D(3)$ we easily obtain

$$
D(3)=d^{3}-d(L R+2 l r)+L r^{2}+R l^{2} .
$$

The recursion of [17] with our notations is

$$
\begin{aligned}
D(n) & =d D(n-1)+(L R-l r) D(n-2)+\left(L r^{2}+R l^{2}-2 d L R\right) D(n-3) \\
& +L R(L R-l r) D(n-4)+d L^{2} R^{2} D(n-5)-L^{3} R^{3} D(n-6) \quad(n=4,5,6, \ldots) .
\end{aligned}
$$


Calculating $D(n)$ for $n=4,5,6,7,8,9$ using (17), (18) with Maple software we obtain

$$
\begin{aligned}
& d^{4}-d^{2}(2 L R+3 l r)+d\left(2 L r^{2}+2 R l^{2}\right)+L^{2} R^{2}-2 L R l r+l^{2} r^{2}, \\
& d^{5}-d^{3}(3 L R+4 l r)+d^{2}\left(3 L r^{2}+3 R l^{2}\right)+d\left(2 L^{2} R^{2}-2 L R l r+3 l^{2} r^{2}\right)+\left(L^{2} R r^{2}+R^{2} L l^{2}-2 L l r^{3}-2 R r l^{3}\right), \\
& d^{6}-d^{4}(4 L R+5 l r)+d^{3}\left(4 L r^{2}+4 R l^{2}\right)+d^{2}\left(4 L^{2} R^{2}+6 l^{2} r^{2}\right)+d\left(-6 L l r^{3}-6 R l^{3} r\right) \\
& +\left(-4 L^{2} R^{2} l r+L^{2} r^{4}+6 L R l^{2} r^{2}+R^{2} l^{4}-l^{3} r^{3}\right), \\
& d^{7}-d^{5}(5 L R+6 l r)+d^{4}\left(5 L r^{2}+5 R l^{2}\right)+d^{3}\left(7 L^{2} R^{2}+4 L R l r+10 l^{2} r^{2}\right) \\
& -d^{2}\left(3 L^{2} R r^{2}+3 R^{2} L l^{2}+12 L l r^{3}+12 R r l^{3}\right)-d\left(2 L^{3} R^{3}+6 L^{2} R^{2} l r-3 L^{2} r^{4}-3 R^{2} l^{4}-15 L R l^{2} r^{2}+4 l^{3} r^{3}\right) \\
& +\left(3 L^{3} R^{2} r^{2}+3 R^{3} L^{2} l^{2}-6 L^{2} R l r^{3}-6 R^{2} L r l^{3}+3 L l^{2} r^{4}+3 R r^{2} l^{4}\right), \\
& d^{8}-d^{6}(6 L R+7 l r)+d^{5}\left(6 L r^{2}+6 R l^{2}\right)+d^{4}\left(11 L^{2} R^{2}+10 L R l r+15 l^{2} r^{2}\right) \\
& +d^{3}\left(-8 L^{2} R r^{2}-8 L R^{2} l^{2}-20 L l r^{3}-20 R l^{3} r\right) \\
& +d^{2}\left(-6 L^{3} R^{3}-9 L^{2} R^{2} l r+6 L^{2} r^{4}+24 L R l^{2} r^{2}+6 R^{2} l^{4}-10 l^{3} r^{3}\right) \\
& +d\left(6 L^{3} R^{2} r^{2}+6 L^{2} R^{3} l^{2}-12 L^{2} R l r^{3}-12 L R^{2} l^{3} r+12 L l^{2} r^{4}+12 R l^{4} r^{2}\right) \\
& +\left(R^{4} L^{4}-6 L^{3} R^{3} l r+2 L^{3} R r^{4}+15 L^{2} R^{2} l^{2} r^{2}-3 L^{2} l r^{5}+2 L R^{3} l^{4}-12 L R l^{3} r^{3}-3 R^{2} l^{5} r+l^{4} r^{4}\right), \\
& d^{9}+(-7 L R-8 l r) d^{7}+\left(7 L r^{2}+7 R l^{2}\right) d^{6}+\left(16 L^{2} R^{2}+18 L R l r+21 l^{2} r^{2}\right) d^{5} \\
& +\left(-15 L^{2} R r^{2}-15 L R^{2} l^{2}-30 L l r^{3}-30 R l^{3} r\right) d^{4} \\
& +\left(-13 L^{3} R^{3}-16 L^{2} R^{2} l r+10 L^{2} r^{4}+30 L R l^{2} r^{2}+10 R^{2} l^{4}-20 l^{3} r^{3}\right) d^{3} \\
& +\left(12 L^{3} R^{2} r^{2}+12 L^{2} R^{3} l^{2}-12 L^{2} R l r^{3}-12 L R^{2} l^{3} r+30 L l^{2} r^{4}+30 R l^{4} r^{2}\right) d^{2} \\
& +\left(3 L^{4} R^{4}-6 L^{3} R^{3} l r+3 L^{3} R r^{4}+30 L^{2} R^{2} l^{2} r^{2}-12 L^{2} l r^{5}+3 L R^{3} l^{4}-44 L R l^{3} r^{3}-12 R^{2} l^{5} r+5 l^{4} r^{4}\right) d \\
& +3 L^{4} R^{3} r^{2}+3 L^{3} R^{4} l^{2}-16 L^{3} R^{2} l r^{3}+L^{3} r^{6}-16 L^{2} R^{3} l^{3} r+18 L^{2} R l^{2} r^{4}+18 L R^{2} l^{4} r^{2}-4 L l^{3} r^{5}+R^{3} l^{6}-4 R l^{5} r^{3} \text {. }
\end{aligned}
$$

Observing these determinants we see that they are monic polynomials

$$
p_{n}(L, l, d, r, R)=d^{n}+\sum_{j=0}^{n-2} A_{n, j}(L, l, r, R) d^{j}
$$

of degree $n$ in $d$ where the coefficients $A_{n, j}(L, l, r, R)(j=0, \ldots, n-2)$ are polynomials of $L, l, r, R$ of degree $n-k$ which are symmetric in $L, R$ and $l, r$. Unfortunately for larger $n$ the formulae for these polynomials are too long. With this we have proved

Theorem 10. Let $1 \leq k<2 k \leq n, n+1=k q+p, 0 \leq p<k$ then for $q=3,4,5,6,7,8$ the determinant of $A_{n+1, k, 2 k}(L, l, d, r, R)$ is

$$
D_{n+1, k, 2 k}(L, l, d, r, R)=p_{q+1}(L, l, d, r, R)^{p} p_{q}(L, l, d, r, R)^{k-p}
$$

where the polynomials $p_{n}$ are given above and by (17).

We conjecture that this theorem is true for all possible values of $q$. 
The imperfect Toeplitz determinants $D^{(\alpha, \beta)}(n+1):=D_{n+1, k, 2 k}^{(\alpha, \beta)}(L, l, d, r, R)$ can be calculated by the recursion

$$
D^{(\alpha, \beta)}(n)=D(n)-(\alpha+\beta) D(n-1)+\alpha \beta D(n-2)
$$

found by Marr and Vineyard [20] and using the previous theorem. Another possibility is to use a nine term recursion based on (19) and (18).

\section{REFERENCES}

[1] N. Bebiano, S. Furtado, A reducing approach for symmetrically sparse banded and anti-banded matrices, Linear Algebra Appl. 581 (2019), 36-50.

[2] U. Brimkulov, Matrices whose inverses are tridiagonal, band or block-tridiagonal and their relationship with the covariance matrices of a random Markov process,Filomat, 33 (2019), no. 5, 1335-1352.

[3] Winston W. Chang, Tai-Liang Chen, Tridiagonal matrices with dominant diagonals and applications,Oper. Res. Lett. 44 (2016), no. 2, 231-233.

[4] E. Egerváry and O. Szász, Einige Extremalprobleme im Bereiche der trigonometrischen Polynome, Math. Z. 27 (1928), 641-652.

[5] C.M. da Fonseca, F.Yilmaz, Some comments on $k$-tridiagonal matrices: determinant, spectra, and inversion, Appl. Math. Comput. 270 (2015), 644-647.

[6] C.M. da Fonseca, V. Kowalenko, Eigenpairs of a family of tridiagonal matrices: three decades later, Acta Math. Hungar. 160 (2) (2020), 376-389.

[7] C.M. da Fonseca, V. Kowalenko, L. Losonczi, Ninety years of k-tridiagonal matrices, Studia Sci. Math. Hung. 57 (3), (2020), 298-311.

[8] C.M. da Fonseca, L. Losonczi, On the determinant of general pentadiagonal matrices, Publ. Math. Debrecen 97/3-4 (2020), 507-523.

[9] C.M. da Fonseca, L. Losonczi, On some pentadiagonal matrices: their determinants and inverses, Annales Univ. Sci. Budapest., Sect. Comp. 51 (2020), 39-50.

[10] A. Frommer, C. Schimmel, M. Schweitzer, Non-Toeplitz decay bounds for inverses of Hermitian positive definite tridiagonal matrices. Electron. Trans. Numer. Anal. 48 (2018), 362-372.

[11] M. El-Mikkawy, F. Atlan, A fast and reliable algorithm for evaluating $n$-th order $k$-tridiagonal determinants, Malaysian J. Math. Sci. 3 (2015), 349-365.

[12] M. El-Mikkawy, T. Sogabe, A new family of $k$-Fibonacci numbers, Appl. Math. Comput. 215 (2010), $4456-4461$.

[13] M. Elouafi, An explicit formula for the determinant of a skew-symmetric pentadiagonal Toeplitz matrix. Appl. Math. Comput. 218 (2011), 3466-3469.

[14] M. Elouafi, On formulae for the determinant of symmetric pentadiagonal Toeplitz matrices. Arab. J. Math. 7 (2018), 91-99.

[15] A.D.A. Hadj, M. Elouafi, On the characteristic polynomial, eigenvectors and determinant of a pentadiagonal matrix. Appl. Math. Comput. 198 (2008), 634-642.

[16] A.D.A. Hadj, M. Elouafi, A fast numerical algorithm for the inverse of a tridiagonal and pentadiagonal matrix. Appl. Math. Comput. 202 (2008), 441-445.

[17] J. Jia, B. Yang, S. Li, On a homogeneous recurrence relation for the determinants of general pentadiagonal Toeplitz matrices, Comput. Math. Appl. 71 (2016), 1036-1044.

[18] L. Losonczi, Eigenvalues and eigenvectors of some tridiagonal matrices. Acta Math. Hung. 60 (1992),309-322.

[19] Y. Lin, X. Lin, A novel algorithm for inverting a $k$-pentadiagonal matrix, The $20163 r d$ International Conference on Systems and Informatics (ICSAI 2016), 578-582.

[20] R.B. Marr, G.H. Vineyard, Five-diagonal Toeplitz determinants and their relation to Chebyshev polynomials, SIAM J. Matrix Anal. Appl. 9 (1988), no.4, 579-586.

[21] T. McMillen, On the eigenvalues of double band matrices, Linear Algebra Appl. 431 (2009), 1890-1897.

[22] A. Ohashi, T. Sogabe, R.S. Usuda, On decomposition of $k$-tridiagonal $\ell$-Toeplitz matrices and its applications, Spec. Matrices 3 (2015), 200-206.

[23] L. Perotti, M. Wojtylak, Matrix methods for Padé approximation: numerical calculation of poles, zeros and residues, Linear Algebra Appl. 548 (2018), 95-122.

[24] R.A. Sweet, A recursive relation for the determinant of a pentadiagonal matrix, Comm. ACM. 12 (1969), $330-332$. 
[25] S. Takahira, T. Sogabe, T.S. Usuda, Bidiagonalization of $(k, k+1)$-tridiagonal matrices, Spec. Matrices 7 (2019), 20-26.

[26] Linda S. L. Tan, Explicit inverse of tridiagonal matrix with applications in autoregressive modelling, IMA J. Appl. Math. 84 (2019), no. 4, 679-695.

[27] C. Wang, H. Li, Di Zhao, An explicit formula for the inverse of a pentadiagonal Toeplitz matrix, J. Comput. Appl. Math. 278 (2015), 12-18.

FACUlTy of ECONOMICS, University of DeBrecen, Hungary

Email address: losonczi08@gmail.com, laszlo.losonczi@econ.unideb.hu 